\title{
Evaluation of donor heart for transplantation
}

\author{
Robert Tatum $^{1}{ }^{10} \cdot$ Alexandros Briasoulis ${ }^{2} \cdot$ Vakhtang Tchantchaleishvili $^{1} \cdot$ H. Todd Massey ${ }^{1}$
}

Accepted: 4 October 2021 / Published online: 4 February 2022

(C) The Author(s), under exclusive licence to Springer Science+Business Media, LLC, part of Springer Nature 2021

\begin{abstract}
Cardiac transplantation is considered the gold-standard treatment option for patients suffering from end-stage heart failure refractory to maximum medical therapy. A major determinant of graft function and recipient survival is a comprehensive evaluation of the donor allograft. Challenges arise when designing and implementing an evidence-based donor evaluation protocol due to the number of influential donor-specific characteristics and the complex interactions that occur between them. Here, we present our systematic approach to donor evaluation by examining the impact that relevant donor variables have on graft function and recipient outcomes.
\end{abstract}

Keywords Heart transplantation $\cdot$ Donor evaluation $\cdot$ Ischemic time $\cdot$ Size-matching

\section{Introduction}

Cardiac transplantation is considered the gold-standard treatment option for patients suffering from end-stage heart failure refractory to maximum medical therapy [1]. Throughout the previous three decades, the relatively stable supply of donor hearts has been insufficient to meet growing recipient demand. The expanding gap between donor supply and recipient need has produced an organ shortage crisis characterized by exorbitant waiting list periods and an unacceptably high waiting list mortality. Ensuring patients have access to this life-saving treatment requires pursuing strategies that not only expand the available donor pool and reduce allograft discard rates, but that also optimize graft function and posttransplant recipient outcomes. Despite the recent rise in total heart transplants due to opioid-related supply increases, the disequilibrium between supply and demand has in fact worsened due to a decrease in donor utilization combined with increasing recipient demand [2]. In healthcare, as elsewhere, scarcity is the mother of allocation, and donor hearts are a finite and highly coveted resource. Scarcity in healthcare requires rationing limited

H. Todd Massey

Howard.Massey@jefferson.edu

Robert Tatum

robtatum91@gmail.com

1 Division of Cardiac Surgery, Department of Surgery, Thomas Jefferson University, 1025 Walnut St, Suite 607, Philadelphia, PA 19107, USA

2 Division of Cardiovascular Medicine, University of Iowa Hospitals and Clinics, 20 Hawkins Drive E318, Iowa City, IA 52242, USA resources through a controlled process of distribution, and the current system unfortunately prioritizes the sickest patients at the expense of taking into account post-transplant prognosis and outcomes [3]. The existing allocation system assumes that scarcity is temporary and that less sick individuals are capable of being rescued in the future [4]. It is imperative that policy making accounts for the significant gains in survival and quality of life achieved through utilization of mechanical circulatory support and recognizes the tremendous impact of a comprehensive donor evaluation on post-transplant recipient outcomes [5]. Previous studies have identified multiple donor characteristics that impact the survival and performance of the transplanted organ [6]. Influential donor factors range from logistical variables (brain death confirmation, verification of consent for donation, $\mathrm{ABO}$ compatibility, geographic constraints, size matching) and comorbid conditions (COPD, coronary artery disease, pulmonary hypertension), to cause of death, laboratory and imaging data, and others [7]. Challenges arise when designing and implementing an evidencebased donor evaluation protocol due to the number of relevant donor-specific characteristics and the complex interactions that occur between them. Here, we present our systematic approach to donor evaluation by examining the impact that relevant donor variables have on graft function and recipient outcomes.

\section{Recipient factors}

Our evaluation of donor hearts begins with the understanding that donor-recipient suitability is highly individualized and dependents on a multitude of factors that interact in 
complex and often unpredictable ways. Baseline demographic variables are the first factors we consider and include recipient age, height, weight, and blood group. Accurate recipient size measurements are crucial to achieving a favorable donor-recipient size match, with donor predicted heart mass playing a more determinative role than weight ratio, body surface area, or body mass index [8].

Risk tolerance for grafts deemed higher risk for primary graft dysfunction is guided by recipient factors such as older age, degree of end organ dysfunction, surgical complexity, and other factors that could potentially limit the implementation of temporary mechanical circulatory support. Increased tolerance for grafts deemed higher risk for primary graft dysfunction is adjudicated in those deemed suitable candidates for post-transplant temporary support. Recipient age impacts our tolerance for transplanting lower quality donors given the negative correlation between recipient age and tolerance for grafts with concerning features.

Next, we perform a thorough medical history with a focus on congenital (septal defects, tetralogy of Fallot, Ebstein anomaly) and acquired (myocardial infarction, pulmonary hypertension, hypertrophic obstructive cardiomyopathy) cardiac pathologies followed by a detailed procedural history. Recipients with severe peripheral vascular disease or uncorrected abdominal aortic aneurysm are rarely candidates for high-risk grafts due to limitations imposed on peripheral support strategies. Individuals with left ventricular assist devices (LVAD) or total artificial heart (TAH) may have extensive mediastinal fibrosis and deleterious thoracic remodeling that can impact donor ischemic times.

Lastly, we evaluate recipient immune system sensitization using calculated panel reaction antibody (cPRA) and mean fluoresent antibody testing. The mortality for sensitized heart transplant patients is increased relative to non-sensitized patients in terms of higher wait-list mortality, antibody mediated rejection with graft dysfunction, and the development of graft vasculopathy $[9,10]$. Although a standardized threshold for determining sensitization has not been established, most centers use a cPRA greater than 50\% and an IgG (MFI) greater than 1000 for defining sensitization.

Our center-specific antibody screening is performed by Luminex solid phase single antigen bead testing for the presence and identification of antibodies to HLA class I (A, $\mathrm{B}, \mathrm{Cw}, \mathrm{Bw}$ ) and class II (DR, DQB1, DRw, DQA1, DPB1) HLA antigens [11]. Based on our institutional protocol those antibodies scoring a MFI of greater than or equal to 5000 will be reported and the cPRA will be calculated based on this threshold. Any antibody attaining the threshold of greater than or equal to 5000 will be tittered and tested for complement fixing utilizing the $\mathrm{C} 1 \mathrm{q}$ assay.

The "cutoff value" for determining a positive MFI is suggestive of "significance" and a semi-quantitative strength of the antibody that would impact the patient's prospective crossmatch. This "cutoff value" is approximate and should not be misinterpreted as guaranteeing safety of the posttransplant outcome. A close working relationship with the institutional immunogenetics and tissue typing laboratory and the clinical transplant team is essential to ensure successful transplantation. The MFI "cutoff value" of avoid antigens can be modified at the discretion of the laboratory and cardiac transplant team taking into account specific data presumed to impact biologic significance such as the ability of the antibody to fix complement. A virtual crossmatch based upon agreed avoid antigens is performed on all recipients with the primary objective to prevent unnecessary prospective crossmatches that would most likely be incompatible. Prospective crossmatch testing is performed by two methods: a serologically-based complement-dependent lymphocytotoxicity assay ( $\mathrm{T}$ cell crossmatch by the standard and antiglobulin method; B cell by the NIH method) and by flow cytometry. Prospective crossmatch is performed in all sensitized patients even if the virtual crossmatch is negative. Desensitization strategies are considered in those with cPRA $>50 \%$ especially in the presence of prior positive cross matches or in the presence of high titer antibodies that fix complement by the C1q assay [12].

\section{Geographic proximity and ischemic time}

Geographic proximity between procurement and transplant locations is the next variable in our assessment due to its impact on organ ischemic time and ultimately on graft performance and recipient survival. In general, there is an inverse relationship between organ ischemic time and posttransplant graft function and recipient survival [13]. For this reason, our optimum graft ischemic time does not exceed 90 min because of the association between prolonged ischemia and allograft vasculopathy and myocardial damage. We do, however, recognize that the magnitude of this impact depends on donor age and the presence of additional risk factors, [14] so we exercise greater tolerance for longer ischemic times in younger donors. We accept grafts with ischemic times that approach 4 hours for young donors with no comorbidities and evidence of good native cardiac function. Although some centers tolerate ischemic times up to $8 \mathrm{~h}$ for optimum donors, there is evidence that such long ischemic times adversely affect early graft performance and recipient survival [15], so we typically classify grafts with ischemic times that exceed $8 \mathrm{~h}$ as unacceptable.

\section{Age}

Cardiac allografts are procured from individuals that span a wide age range [16], and increased donor age is a significant risk factor for posttransplant morbidities including coronary artery disease, allograft vasculopathy, and need for 
retransplantation. For this reason, we recognize the International Society for Heart and Lung Transplantation (ISHLT) designation of donor age as an independent predictor of early cardiac graft failure and use donor age of 60 as our upper limit of acceptability. The majority of allografts we accept are from donors between 18 and 40 years old because of more favorable outcomes on early graft performance and long-term survival [17].

Most studies examining the impact of donor age on recipient outcomes have found an inverse relationship between donor age and recipient posttransplant survival, particularly among middle and older-age recipients [18]. This association remains after controlling for 90-day mortality, suggesting that the mechanism of donor age impacting recipient survival goes beyond its effect on early graft dysfunction. The negative association between donor age and recipient survival is incompletely understood but is likely multifactorial in nature. Proposed mechanisms include greater sensitivity to ischemia, increased myocardial fibrosis, atherosclerotic vascular disease, and valvular thickening. The increased mortality risk is closely linked to this array of degenerative changes, so conducting a thorough functional assessment requires correlating patient age with cardiac performance on echocardiogram.

Lastly, our donor assessment acknowledges that the elevated risk of utilizing hearts from older donors must be weighed against non-transplant due to lack of a younger donor. The strategic utilization of older donor hearts to expand the donor pool is plagued by a lack of sufficient data to guide clinical decision-making. Differing risk mitigation strategies to increase older donor heart utilization include matching older donors to older recipients to theoretically optimize the potential of better aligning long term outcomes to recipient age [19]. A contemporary retrospective review of the UNOS database for all first-time adult heart transplant recipients between 2005 and 2018 analyzed the interaction between donor and recipient age and concluded that the "cost" of an older donor in heart transplantation is unchanged by recipient age and is not mitigated by using a younger recipient [20]. Similarly, the adverse effect of an older recipient is not modified by the age of the donor. We typically avoid utilizing older donor hearts in the younger recipients given the negative effects on short and long term outcomes except in extreme circumstances.

\section{Cause of death}

Donor cause of death influences allograft rejection and recipient survival [21]. Despite the present interest in donation after cardiac death (DCD) donors, donation after brain death (DBD) donors remain the primary contributors to heart transplants and account for the overwhelming majority of grafts we accept. Optimum DBD donors are young individuals with no medical comorbidities or surgical risk factors. They are often victims of motor vehicle accidents or gun-shot wounds, provided the traumatic mechanism avoided damage to cardiac structures. Other causes of mortality for DBD donors include stroke, anoxic encephalopathy from asphyxiation (drowning, drug overdose such as opioids, chemical asphyxiates such as carbon monoxide, anaphylaxis, choking, strangulation, hanging, asthmatic attacks, seizures, cardiorespiratory arrest), brain tumors, infections such as encephalitis, or occasionally death from an unknown etiology [22]. A complete evaluation of the cause of brain death is warranted to avoid any potential implications for the recipient [23]. Potential donors who suffer intracranial hemorrhage require thorough investigation into the underlying cardiovascular pathology and careful tracking of graft performance. Individuals who suffer aneurysmal rupture and display adequate or improving cardiac function may be acceptable candidates, whereas potential donors who suffer brain hemorrhage associated with severe hypertension with evidence of ventricular hypertrophy are considered higher risk for primary graft dysfunction.

Donor hearts from poisoning such as those associated with carbon monoxide inhalation require extra discrimination in assessing the potential of the specific toxin to cause acute as well as chronic cardiac dysfunction. Carbon monoxide in particular reduces oxygen delivery by competitively binding to hemoglobin and subsequently reducing oxygen delivery to tissues [24]. The effects of carbon monoxide poisoning can lead to cardiac enzyme leak, acute myocardial dysfunction, and an increased long-term risk of developing coronary disease and various potentially fatal arrhythmias. When considering utilization of donor allografts from patients dying of drug and poison intoxication, a comprehensive understanding of the toxicology and clinical implications is paramount. The literature in organ transplantation regarding short and long term outcomes utilizing donor hearts from fatal drug intoxication and poisoning remains sparse. For this reason consultation with experts in toxicology is warranted.

Donor hearts from drug overdoses have previously been underutilized based on concerns for donor quality and the possibility of increased transmission of infectious diseases [25]. The opioid epidemic has significantly increased the supply of donor organs available for transplantation and multiple studies have shown outcomes of transplants from donors who died from drug-related causes are comparable to transplants from donors who died due to other mechanisms [26].

\section{Donor history}

We examine the presence and severity of donor medical comorbidities thoroughly before making determinations 
about graft utilization and recipient suitability. Common donor comorbidities that influence graft function and longterm recipient survival include hypertension, smoking history, prior cocaine use, chronic obstructive pulmonary disease, and a history of cardiac arrest [27]. We believe carefully selected diabetic donors are acceptable and appear to carry no additional risk for early graft dysfunction or posttransplant mortality compared to matched controls without insulin dysfunction [28]. Donors with active or recently treated malignancy are invariably avoided due to the risk of malignancy transmission and associated poor outcomes. The risk of utilizing donors with primary brain tumors remains controversial, especially in regards to those with high grade gliomas and medullablastomas [29]. The risk of transmission is considered greater if the blood brain barrier has been breached through surgical intervention, radiotherapy, or ventriculosystemic shunt placement. Most transplant centers prohibit the utilization of donor grafts with significant coronary artery disease due to increased rates of primary graft dysfunction (PGD) and recipient need for retransplantation. Selective use of donor hearts with mild or moderate coronary artery disease with excellent function on echocardiogram may be acceptable, although the increased demand for revascularization with angioplasty or recipient conduit in these patients often prohibits their utilization [30]. Our tolerance for donor coronary artery disease is based on the remaining donor risk profile and recipient functional status. Individuals with an extensive substance abuse history are avoided, particularly those abusing stimulants and other noradrenergic agents.

\section{Size-matching}

The fundamental goal of cardiac size-matching is to minimize morphological discrepancies between donor and recipient that may negatively affect postoperative cardiac function, a phenomenon we notice most often in recipients of undersized donor hearts [31]. This observation is due to a constellation of physiologic disadvantages placed on the transplanted heart that worsen with under sizing. The transplanted heart experiences early chronotropic incompetence which requires augmentation of cardiac workflow to allow for sustained systemic perfusion. This is accomplished predominantly through increasing stroke volume, a hemodynamic adaptation that requires chronically increased filling pressures that can have particularly damaging effects on recipients of undersized donor hearts [32].

Although there is currently no standardized sizing metric, predicted heart mass (PHM) is our preferred tool based on institutional experience combined with an array of confirmational studies $[33,34]$. We also recognize ISHLT guidelines which recommend the use of a donor male whose body weight is no less than $30 \%$ that of the recipient, and no less than $20 \%$ below if the donor is female [35]. Cardiac mass calculations are specifically useful for taking into account the weighted contributions of age, sex, weight, and height in size matching. We prefer to limit under sizing of the donor in most heart failure patients to less than $-15 \%$ of cardiac mass due to an association with increased post-transplant mortality. Tolerance for oversizing is greater and in some instances preferred in recipients with pulmonary hypertension, longer donor ischemic times, older donor hearts, and donor hearts with cardiac dysfunction. Avoidance of undersized donors is particularly crucial in recipients with subtantial pulmonary hypertension to prevent acute and chronic right ventricular dysfunction and to avoid the associated increased mortality risk.

Oversizing is not without risk, however, as previously published work has shown that excessive oversizing can, in extremes, lead to increased mortality, likely attributable to restrictive physiology. The limit for excessive oversizing is based on two standard deviations of height being six inches for men and five inches for women. There are higher risk circumstances where the oversizing limit would be restricted to one standard deviation, such as in patients previously receiving urgent LVAD after acute myocardial infarction without remodeling, chronic Syncardia TAH support without tissue expanders, non-dilated restrictive cardiomyopathies, radiation induced cardiomyopathy, or multiple prior cardiac surgeries without native heart dilatation or other potential pericardial space-restricting conditions [36]. We have found it useful to use recipients' cardiothoracic ratio (CTR) to determine the extent of cardiomegaly and thereby approximately estimate the available space in the chest for a donor heart [37]. As such, CTR could be useful in defining the limits of oversizing.

\section{Infections}

We regularly screen donors for the presence and risk of infections via serologic testing, blood and urine cultures, comprehensive social and medical histories, and chest X-ray. Donorderived infections in transplantation have been described for a wide range of pathogens inclusive of viral, bacterial, mycobacterial, fungal and parasitic disease with significant risk of morbidity and mortality approaching $30 \%$ [38].

The ISHLT recommends a thorough graft inspection to exclude the possibility of donor endocarditis because of associated poor recipient outcomes [39]. Other ISHLT recommendations regarding donor infections that we rigorously follow include the exclusion of sepsis-induced myocardial dysfunction, avoidance of donors who died greater than $96 \mathrm{~h}$ after hospital admission, and obtaining consecutive negative blood cultures prior to procurement [40]. In our practice donors with bacteremia can be considered if pathogen specific antibiotics have been administered for at least 48 hours prior to procurement with some degree of clinical response, and there has been a complete echocardiographic assessment 
of the valves to exclude endocarditis. Patients with positive blood cultures for fungal, atypical bacterial, or multi-drug resistant bacterial cultures are not utilized. Other unsuitable candidates include those with undiagnosed febrile illness, encephalitis, meningitis or those with flaccid paralysis of unknown etiology. We avoid potential donors who test positive for syphilis, Mycobacterium tuberculosis, non-TB mycobacterium, and various fungal infections such as aspergillus or cryptococcus.

Absolute contraindications to donation are infections with HIV, human T-cell leukemia-lymphoma virus, systemic viral infections (e.g., measles, rabies, adenovirus, enterovirus, and parvovirus), prion-related disease, and herpetic meningoencephalitis [41].The Organ Procurement and Transplantation Network (OPTN) policy requires screening donors for syphilis, hepatitis B/C, HIV, cytomegalovirus (CMV), and Epstein-Barr virus (EBV) [43]. Although historically unacceptable, we implant grafts from donors with serologic evidence of hepatitis $\mathrm{C}$ virus infections due to remarkably effective antiviral therapies and clinical evidence of comparable outcomes to non-infectious donors [42]. Transmission risks vary significantly based on the pathogen inoculum and immunosuppressive regimen, and recipients should be informed that no screening test can eliminate the possibility of transmission [44].

The risk of developing COVID-19 from a SARS-CoV-2 infected donor organ is unknown [45]. Current OPTN and CDC guidance on deceased donor screening recommends testing deceased donors for SARS-CoV-2 by nucleic acid testing (NAT) from a respiratory sample within 72 hours of donation [46]. For individuals with resolved infection and negative NAT at the time of donor evaluation, the risk of infectious transmission is extraordinarily low [47].

\section{Hemodynamic management and inotrope requirements}

Brain death can lead to significant hemodynamic, hormonal, inflammatory and metabolic disturbances that can have negative effects on donor organ quality. These effects can vary based upon whether the inciting event precipitates an acute massive increase in intracranial pressure (ICP) versus mortality associated with a gradual increase in ICP [48]. Brainstem death related to a progressive rise in ICP leads to sequential loss of function of the midbrain, the pons and ultimately the medulla oblongata with severe pathophysiologic perturbations due to imbalances, surges, and depletion of neurochemical transmitters vital to the autonomic nervous system [49].

The progressive ischemia and death of autonomic regulatory nuclei within the brainstem manifest clinically with an initial parasympathetic stimulation with resultant apnea, hypotension and bradycardia followed by a period known as the classic Cushing's reflex, manifested by hypertension and bradycardia [50]. This state progresses to loss of the vagal cardiomotor nuclei, leading to unopposed sympathetic stimulation (autonomic storm), ultimately culminating in sympathetic depletion and loss of vasomotor tone and development of neurogenic shock. This period of autonomic storm can be especially damaging in regards to cardiac function due to neurogenic catecholamine cardiotoxicity with myocardial ischemia and necrosis that appears directly related to the rate of rise of ICP and can be blocked by pharmacologic sympathectomy. This autonomic dysregulation is accompanied by dysregulation of the hypophyseal-pituitaryadrenocorticoid axis, activation of the systemic inflammatory response, loss of thermoregulatory control, alterations in coagulation with possible development of disseminated intravascular coagulation, and profound metabolic derangements. Prolonged cardiovascular instability at the time of death is associated with decreased allograft utilization and increased organ discard. There remains much debate regarding the management of the brain dead donor in terms of who manages the donor, the type of volume resuscitation, hemodynamic and metabolic targets, hormonal resuscitation, usage of catecholamine and vasopressor agents, requirements of invasive and noninvasive testing of specific organs, and timing and conduct of organ procurement. The vast majority of evidence favoring one donor management method over another is based on observational studies, individual expert opinion, and various society consensus guidelines with few prospective studies to guide protocol design. Given the expanding deficit in donor supply for all organs, the emphasis should remain on increasing the net yield of all organs per donor in conjunction with expanding the donor pool for all organs.

Our institutional approach to donor management goals is pragmatic, similar to that for any critically ill patient, and is designed to optimize and maintain organ function by focusing on maintaining euvolemia, hemodynamic stability, and adequate tissue oxygenation. Our program donor management protocols have foundational principles based upon the original recommendations set forth by the Crystal City Cardiac Consensus Conference report [51].

The initial conventional approach to the heart donor should include maintenance of normal perfusion pressures with target MAP > 65 with limited utilization of inotropes and vasopressors. Diligent correction of any metabolic abnormalities, hypoxemia, hypercarbia, and acid-base abnormalities should be undertaken. After optimization of hemodynamics, a thorough echocardiogram should be obtained for the initial assessment of cardiac function and for delineation of any structural abnormalities. If the initial assessment reveals significant myocardial dysfunction $(\mathrm{EF}<45 \%)$, an additional period of cardiac resuscitative donor management should commence, utilizing neuroendocrine replacement 
therapy with invasive hemodynamic monitoring and delayed (usually $>$ six hours) echocardiogram re-imaging [52].

\section{Laboratory studies}

A thorough laboratory evaluation is performed to exclude multiorgan damage and examine the potential for myocardial injury. Troponin and CPK-MB are biomarkers of myocardial cell damage, and investigations into their prognostic value on recipient outcomes have shown mixed results. Some studies suggest elevated troponin levels may be correlated with poor graft function [53], although newer studies on larger populations have failed to confirm these results [54]. We regularly draw troponin levels to evaluate for graft dysfunction, but mildly increased values are not, in isolation, prohibitive to donor utilization, particularly in conjunction with favorable electrocardiographic and echocardiographic findings. Elevated B-type natriuretic peptide (BNP) levels are a reliable indicator of reduced cardiac output after transplantation, and we rarely transplant recipients from donors with BNP $>160 \mathrm{pg} / \mathrm{mL}$ because of the associated risk for postoperative poor cardiac function (cardiac index $<2.2 \mathrm{~L} / \mathrm{min} / \mathrm{m}^{2}$ at day 12) [55]. The concentrations of various inflammatory markers also provide prognostic information, including procalcitonin, tumor necrosis factor-alpha (TNF- $\alpha$ ), and interleukins 1 and 6. Elevated procalcitonin levels are associated with increased early graft failure, and increased (TNF- $\alpha$ ) concentrations correlate with reduced hemodynamic status of the donor. BUN/Cr is associated with decreased graft survival 1 year posttransplant and is included in our donor risk assessment. Currently, no biomarker in isolation can be used to reliably classify a donor heart as appropriate for transplant. All lab values must be correlated with echocardiographic findings to determine their prognostic significance.

\section{Echocardiography}

A complete transthoracic echocardiogram (TTE) is performed as part of every donor assessment to evaluate graft characteristics and functional performance. TTE allows for a comprehensive morphological and functional assessment that carries predictive significance for short-term graft function and both short-and-moderate-term recipient mortality. Echocardiographic parameters that we examine closely include cardiac size, left-ventricular systolic function, regional wall motion abnormalities, ventricular hypertrophy, septal defects, valve integrity, and coronary flow. All echocardiograms and diagnostic studies pertaining to individual donors are reviewed by specialized team members prior to formal acceptance or denial. In select circumstances a team member with echocardiographic skills will travel with the procurement team for on-site evaluation. Typically, grafts with an ejection fraction (EF) greater than $50 \%$ are considered acceptable for transplantation [56]. Although reduced donor EF correlates with increased rates of non-use, 1-year survival is comparable to recipients of reduced EF donors. We consider grafts that have depressed EF for transplantation provided the donor undergoes a period of aggressive management with hemodynamic monitoring and serial echocardiographic assessment to guide therapy that results in normalization of function [57].

\section{Cardiac catheterization and angiography}

Invasive hemodynamic monitoring allows for a comprehensive morphological and functional assessment that carries predictive significance for short-term graft function and both short-and-moderate-term recipient mortality. Right heart catherization is rarely performed unless specifically indicated to obtain invasive hemodynamic monitoring in patients undergoing aggressive donor management for potentially reversible cardiac dysfunction or in the assessment of particular cardiac anomalies. In hemodynamically labile donors, invasive hemodynamic monitoring via cardiac catheterization can potentially guide therapy and increase utilization of grafts that traditionally would be disqualified. Optimum donor hemodynamic values include a central venous pressure (CVP) 8-12 mmHg, pulmonary capillary wedge pressure $<14 \mathrm{mmHg}$, mean arterial pressure (MAP) $>65 \mathrm{mmHg}$, and left ventricular stroke work index $>15 \times \mathrm{gm} / \mathrm{m}$ [58]. Recipient tolerance for suboptimal values varies based on concomitant inotropic dependence, ischemic time, and donor age. Angiography is another important component of our donor assessment that permits visualization of coronary flow and myocardial perfusion. We routinely require coronary angiogram for all donors over 40 years, as well as younger donors at risk for coronary disease. While existence of a flow-limiting lesion ( $>50 \%$ stenosis) without revascularization plan would preclude utilization of the donor heart, revascularization at the time of heart transplant does not mitigate the risk of an ischemic event during the procurement. Lower grade focal lesions do not preclude the donor heart use but long-term outcomes are not clear due to paucity of the data in the literature. Additional angiographic findings such as coronary spasm and myocardial bridge should also be factored in very carefully and correlated with additional donor information, in particular substance use such as methamphetamine and cocaine.

\section{Machine perfusion assessment}

Ex situ machine perfusion (MP) is an exciting frontier in donor assessment. Although not currently part of our program's algorithm, we acknowledge the tremendous potential of these devices to facilitate the use of extended criteria donors and promote organ recovery through therapeutic drug and nutri- 
ent delivery [59]. Normothermic machine perfusion permits visual inspection of contractile function and coronary blood flow under conditions that mimic human physiology, a capacity with potentially high importance considering evaluations of left ventricular contractility using machine perfusion are strongly correlated with posttransplant cardiac function [60]. Measuring hemodynamic parameters in a physiologic environment allows for a detailed circulatory assessment prior to transplantation. Future research investments should prioritize standardizing perfusion conditions and improving our understanding of which biomarkers can reliably predict graft function and quality.

\section{Conclusion}

Ensuring that available grafts are equitably distributed to waitlist candidates requires a thorough and detailed assessment of organ allocation parameters and algorithms. Various approaches have been proposed and significant disagreement exists regarding the optimum strategy for achieving a fair and ethical means of organ distribution. The cardiac allocation score (CAS) was developed as an analogous scheme to the Lung Allocation Score (LAS) that determines urgency based on a consortium of relevant demographic and clinical recipient factors [61]. Other approaches have incorporated waitlist mortality and heavily prioritize posttransplant prognosis. Achieving a reliable, standardized cardiac allocation system will require pursuing functional assessment tools and laboratory information that minimize the possibility for bias and prioritize objective data points. The left ventricular end-systolic pressure volume relationship (ESPVR) has been proposed as a load-independent determinant of left ventricular function for patients with systolic heart failure that could prove valuable in assessing recipient functional performance and ultimately candidate urgency [62]. The use of NT-ProBNP has also been suggested as a useful predictor of adverse events for hospitalized patients suffering from either systolic or diastolic heart failure as one component of an objective cardiac allocation system [63]. There may be a role for machine learning algorithms in determining severity of recipient need, although systems at present require additional layers of sophistication in order to perform a comprehensive and reliable recipient assessment [64]. Irrespective of the precise components of such a system, allocation scoring should be vigorously pursued in a way that seeks to minimize the possibility for subjectivity and ensures an impartial distribution of this life-saving and finite resource.

\section{References}

1. Alraies MC, Eckman P (2014) Adult heart transplant: indications and outcomes. J Thorac Dis. https://doi.org/10.3978/j.issn.20721439.2014.06.44
2. Mancini D, Lietz K (2010) Selection of cardiac transplantation candidates in 2010. Circulation. https://doi.org/10.1161/ CIRCULATIONAHA.109.858076

3. Colvin-Adams M, Valapour M, Hertz M, Heubner B, Paulson K, Dhungel V, Skeans MA, Edwards L, Ghimire V, Waller C, Cherikh WS, Kasiske BL, Snyder JJ, Israni AK. Lung and heart allocation in the United States. Am J Transplant. 2012;12(12):3213-3234. https://doi.org/10.1111/j.1600-6143. 2012.04258.x

4. Persad G, Wertheimer A, Emanuel EJ. Principles for allocation of scarce medical interventions. Lancet. 2009. https://doi.org/10. 1016/S0140-6736(09)60137-9

5. Estep J, Soltesz E, Cogswell R. The new heart transplant allocation system: Early observations and mechanical circulatory support considerations. J Thorac Cardiovasc Surg. 2020;S00225223(20)32638-6. https://doi.org/10.1016/j.jtcvs.2020.08.113

6. Mullan CW, Sen S, Ahmad T. Left Ventricular Assist Devices Versus Heart Transplantation for End Stage Heart Failure is a Misleading Equivalency. JACC Hear Fail. 2021. https://doi.org/ 10.1016/j.jchf.2021.02.004

7. Latif F, Farr MA, Clerkin KJ, et al. Characteristics and Outcomes of Recipients of Heart Transplant with Coronavirus Disease 2019. JAMA Cardiol. 2020. https://doi.org/10.1001/jamacardio.2020. 2159

8. Khush KK, Cherikh WS, Chambers DC, et al. The International Thoracic Organ Transplant Registry of the International Society for Heart and Lung Transplantation: Thirty-sixth adult heart transplantation report - 2019; focus theme: Donor and recipient size match. J Hear Lung Transplant. 2019. https://doi.org/10.1016/j.healun.2019. 08.004

9. Colvin MM, Cook JL, Chang PP, et al. Sensitization in Heart Transplantation: Emerging Knowledge: A Scientific Statement From the American Heart Association. Circulation. 2019. https://doi.org/10. 1161/CIR.0000000000000598

10. Barten MJ, Schulz U, Beiras-Fernandez A, et al. The clinical impact of donor-specific antibodies in heart transplantation. Transplant Rev. 2018. https://doi.org/10.1016/j.trre.2018.05.002

11. Deo S V., Al-Kindi SG, Altarabsheh SE, et al. Model for end-stage liver disease excluding international normalized ratio (MELD-XI) score predicts heart transplant outcomes: Evidence from the registry of the United Network for Organ Sharing. J Hear Lung Transplant. 2016. https://doi.org/10.1016/j.healun.2015.10.008

12. Chih S, Patel J. Desensitization strategies in adult heart transplantation-Will persistence pay off? J Hear Lung Transplant. 2016. https://doi.org/10.1016/j.healun.2016.03.021

13. Russo MJ, Chen JM, Sorabella RA, et al. The effect of ischemic time on survival after heart transplantation varies by donor age: An analysis of the United Network for Organ Sharing database. J Thorac Cardiovasc Surg. 2007. https:// doi.org/10.1016/j.jtcvs.2006.09.019

14. Buendía-Fuentes F, Almenar-Bonet L, Martínez-Dolz L, et al. Ischemic Time as a Predictor of Physical Recovery in the First Months after Heart Transplantation. ISRN Cardiol. 2012. https:// doi.org/10.5402/2012/907102

15. Scheule AM, Zimmerman GJ, Johnston JK, Razzouk AJ, Gundry SR, Bailey LL. Duration of graft cold ischemia does not affect outcomes in pediatric heart transplant recipients. Circulation. 2002. https://doi.org/10.1161/01.cir.0000032882.55215.00

16. Chamogeorgakis $\mathrm{T}$, Joseph $\mathrm{S}$, Hall $\mathrm{S}$, et al. Impact of donor age on cardiac transplantation outcomes and on cardiac function. Interact Cardiovasc Thorac Surg. 2016. https://doi.org/10.1093/ icvts/ivw 172

17. Sorabella RA, Guglielmetti L, Kantor A, et al. Cardiac Donor Risk Factors Predictive of Short-Term Heart Transplant 
Recipient Mortality: An Analysis of the United Network for Organ Sharing Database. Transplant Proc. 2015. https://doi.org/ 10.1016/j.transproceed.2015.10.021

18. Gupta D, Piacentino V, MacHa M, et al. Effect of older donor age on risk for mortality after heart transplantation. Ann Thorac Surg. 2004. https://doi.org/10.1016/j.athoracsur.2004.02.016

19. Ram E, Lavee J, Kogan A, et al. Does donor-recipient age difference matter in outcome of heart transplantation? Clin Transplant. 2019. https://doi.org/10.1111/ctr.13593

20. Kilic A, Allen JG, Weiss ES. Validation of the United Statesderived Index for Mortality Prediction after Cardiac Transplantation (IMPACT) using international registry data. J Hear Lung Transplant. 2013. https://doi.org/10.1016/j.healun.2013.02.001

21. Singhal AK, Sheng X, Drakos SG, Stehlik J. Impact of Donor Cause of Death on Transplant Outcomes: UNOS Registry Analysis. Transplant Proc. 2009. https://doi.org/10.1016/j.transproceed. 2009.06.192

22. Martìn-Suàrez S, Mikus E, Pilato E, et al. Cardiac Transplantation From a Carbon Monoxide Intoxicated Donor. Transplant Proc. 2008. https://doi.org/10.1016/j.transproceed.2008.03.155

23. Cohen O, De La Zerda DJ, Beygui R, Hekmat D, Laks H. Donor Brain Death Mechanisms and Outcomes After Heart Transplantation. Transplant Proc. 2007. https://doi.org/10.1016/j.transproceed. 2007.08.102

24. Guzman JA. Carbon Monoxide Poisoning. Crit Care Clin. 2012. https://doi.org/10.1016/j.ccc.2012.07.007

25. Altshuler PJ, Helmers MR, Atluri P. Organ allocation and procurement in cardiac transplantation. Curr Opin Organ Transplant. 2021. https://doi.org/10.1097/MOT.0000000000000872

26. Phillips KG, Ranganath NK, Malas J, et al. Impact of the Opioid Epidemic on Heart Transplantation: Donor Characteristics and Organ Discard. Ann Thorac Surg. 2019. https://doi.org/10.1016/j. athoracsur.2019.03.076

27. Guihaire J, Noly PE, Martin A, et al. Impact of donor comorbidities on heart transplant outcomes in the modern era. Interact Cardiovasc Thorac Surg. 2017. https://doi.org/10.1093/icvts/ivx014

28. Taghavi S, Jayarajan SN, Wilson LM, Komaroff E, Testani JM, Mangi AA. Cardiac transplantation can be safely performed using selected diabetic donors. J Thorac Cardiovasc Surg. 2013. https:// doi.org/10.1016/j.jtcvs.2013.02.047

29. Huang S, Tang Y, Zhu Z, et al. Outcomes of organ transplantation from donors with a cancer history. Med Sci Monit. 2018. https:// doi.org/10.12659/MSM.909059

30. Kimura Y, Seguchi O, Iwasaki K, et al. Impact of coronary artery calcification in the donor heart on transmitted coronary artery disease in heart transplant recipients. Circ J. 2018. https://doi.org/ 10.1253/circj.CJ-18-0107

31. Ziaziaris W, Chew HC, Dhital K, Hayward C, Pleass H, Macdonald P. Size and Gender Matching in Heart Transplantation - Optimizing Donor Utilization in an Era of Changing Donor and Recipient Characteristics. Curr Transplant Reports. 2014. https://doi. org/10.1007/s40472-014-0032-7

32. Reed RM, Netzer G, Hunsicker L, et al. Cardiac Size and SexMatching in Heart Transplantation: Size Matters in Matters of Sex and the Heart. JACC Hear Fail. 2014. https://doi.org/10.1016/j. jchf.2013.09.005

33. Tremblay-Gravel M, Khush K. Predicted Heart Mass for Donor Organ Allocation. Circ Hear Fail. 2019. https://doi.org/10.1161/ circheartfailure.119.006634

34. Cheshire C, Kydd A, Nerlekar N, et al. Size matching in heart transplantation: Is predicted heart mass the optimal method in a United Kingdom cohort? Clin Transplant. 2021. https://doi.org/ 10.1111/ctr.14192

35. Costanzo MR, Dipchand A, Starling R, et al. The international society of heart and lung transplantation guidelines for the care of heart transplant recipients. J Hear Lung Transplant. 2010. https:// doi.org/10.1016/j.healun.2010.05.034

36. Fischer SA, Avery RK. Screening of donor and recipient prior to solid organ transplantation. Am J Transplant. 2009. https://doi. org/10.1111/j.1600-6143.2009.02888.x

37. Sukhavasi A, Weber MP, Maynes EJ, Brailovsky Y, Shah MK, Rajapreyar I, Alvarez RJ, Rame JE, Entwistle JW, Massey HT, Tchantchaleishvili V. Utility of Recipient Cardiothoracic Ratio in Predicting Delayed Chest Closure after Heart Transplantation. International Society for Heart and Lung Transplantation 41st Annual Meeting and Scientific Sessions. 2021.

38. Ison MG, Nalesnik MA. An update on donor-derived disease transmission in organ transplantation. Am J Transplant. 2011. https://doi.org/10.1111/j.1600-6143.2011.03493.x

39. White SL, Rawlinson W, Boan P, et al. Infectious disease transmission in solid organ transplantation: Donor evaluation, recipient risk, and outcomes of transmission. Transplant Direct. 2019. https://doi.org/10.1097/TXD.0000000000000852

40. Dorent R, Gandjbakhch E, Goéminne C, et al. Assessment of potential heart donors: A statement from the French heart transplant community. Arch Cardiovasc Dis. 2018. https://doi.org/10. 1016/j.acvd.2017.12.001

41. Westphal GA, Garcia VD, De Souza RL, et al. Guidelines for the assessment and acceptance of potential brain-dead organ donors. Rev Bras Ter Intensiva. 2016. https://doi.org/10.5935/0103-507X. 20160049

42. Botha J, Fabian J, Etheredge H, Conradie F, Tiemessen CT (2019) HIV and solid organ transplantation: where are we now. Curr HIV/AIDS Rep. https://doi.org/10.1007/s11904-019-00460-7

43. Blumberg EA. Organs from Hepatitis C Virus-Positive Donors. N Engl J Med. 2019. https://doi.org/10.1056/nejme1901957

44. Huprikar S, Danziger-Isakov L, Ahn J, et al. Solid organ transplantation from hepatitis B virus-positive donors: Consensus guidelines for recipient management. Am J Transplant. 2015. https:// doi.org/10.1111/ajt.13187

45. Shah MB, Lynch RJ, El-Haddad H, Doby B, Brockmeier D, Goldberg DS. Utilization of deceased donors during a pandemic: argument against using SARS-CoV-2-positive donors. Am J Transplant. 2020. https://doi.org/10.1111/ajt.15969

46. NasrAllah MM, Osman NA, Elalfy M, Malvezzi P, Rostaing L. Transplantation in the era of the Covid-19 pandemic: How should transplant patients and programs be handled? Rev Med Virol. 2021. https://doi.org/10.1002/rmv.2149

47. Weiss MJ, Lalani J, Patriquin-Stoner C, et al. Summary of International Recommendations for Donation and Transplantation Programs during the Coronavirus Disease Pandemic. Transplantation. 2021. https://doi.org/10.1097/TP.0000000000003520

48. Watson CJ, Roberts R, Wright KA, Greenberg DC, Rous BA, Brown CH, Counter C, Collett D, Bradley JA. How safe is it to transplant organs from deceased donors with primary intracranial malignancy? An analysis of UK Registry data. Am J Transplant. 2010;10(6):1437-44. https://doi.org/10.1111/j.1600-6143.2010. 03130. $\mathrm{x}$

49. Tanim Anwar ASM, Lee JM. Medical management of brain-dead organ donors. Acute Crit Care. 2019. https://doi.org/10.4266/ acc. 2019.00430

50. Kotloff RM, Blosser S, Fulda GJ, et al. Management of the Potential Organ Donor in the ICU. Crit Care Med. 2015. https://doi.org/ 10.1097/ccm.0000000000000958

51. Rosengard BR, Feng S, Alfrey EJ, Zaroff JG, Emond JC, Henry ML, Garrity ER, Roberts JP, Wynn JJ, Metzger RA, Freeman RB, Port FK, Merion RM, Love RB, Busuttil RW, Delmonico FL. Report of the Crystal City meeting to maximize the use of organs recovered from the cadaver donor. Am J Transplant. 2002;2(8):701-11. https://doi.org/10.1034/j.1600-6143.2002. 20804.x 
52. Yang HY, Lin CY, Tsai YT, Lee CY, Tsai CS. Experience of heart transplantation from hemodynamically unstable brain-dead donors with extracorporeal support. Clin Transplant. 2012. https://doi. org/10.1111/j.1399-0012.2011.01585.x

53. Potapov EV, Ivanitskaia EA, Loebe M, et al. Value of cardiac troponin I and $\mathrm{T}$ for selection of heart donors and as predictors of early graft failure. Transplantation. 2001. https://doi.org/10.1097/ 00007890-200105270-00007

54. Madan S, Saeed O, Shin J, et al. Donor Troponin and Survival after Cardiac Transplantation: An Analysis of the United Network of Organ Sharing Registry. Circ Hear Fail. 2016. https://doi.org/ 10.1161/CIRCHEARTFAILURE.115.002909

55. Vorlat A, Conraads VM, Jorens PG, et al. Donor B-type natriuretic peptide predicts early cardiac performance after heart transplantation. J Hear Lung Transplant. 2012. https://doi.org/10.1016/j. healun.2012.02.009

56. Chen CW, Sprys MH, Gaffey AC, et al. Low ejection fraction in donor hearts is not directly associated with increased recipient mortality. J Hear Lung Transplant. 2017. https://doi.org/10.1016/j. healun.2017.02.001

57. Forni A, Luciani GB, Chiominto B, Pilati M, Mazzucco A, Faggian G. Impact of donor quality on outcome of heart transplantation. Eur J Cardio-thoracic Surg. 2010. https://doi.org/10.1016/j. ejcts.2010.03.042

58. Stoica SC, Satchithananda DK, Charman S, et al. Swan-Ganz catheter assessment of donor hearts: Outcome of organs with borderline hemodynamics. J Hear Lung Transplant. 2002. https://doi. org/10.1016/S1053-2498(02)00380-7
59. Bona M, Wyss RK, Arnold M, et al. Cardiac graft assessment in the era of machine perfusion: Current and future biomarkers. J Am Heart Assoc. 2021. https://doi.org/10.1161/JAHA.120. 018966

60. Ribeiro RVP, Alvarez JS, Yu F, et al. Comparing Donor Heart Assessment Strategies during Ex Situ Heart Perfusion to Better Estimate Posttransplant Cardiac Function. Transplantation. 2020. https://doi.org/10.1097/TP.0000000000003374

61. Claes S, Berchtold-Herz M, Zhou Q, et al. Towards a cardiac allocation score: A retrospective calculation for 73 patients from a German transplant center. J Cardiothorac Surg. 2017. https://doi.org/10.1186/s13019-017-0575-7

62. Kohli K, Kovács SJ. The quest for load-independent left ventricular chamber properties: exploring the normalized pressurevolume loop. Physiol Rep. 2017. https://doi.org/10.14814/phy2. 13160

63. Wang Y, Zhang R, Huang Y, et al. Combining the use of aminoterminal pro-B-type natriuretic peptide and B-type natriuretic peptide in the prognosis of hospitalized heart failure patients. Clin Chim Acta. 2019. https://doi.org/10.1016/j.cca.2018.12.025

64. Zahavi G, Frogel J, Shlomo N, Klempfner R, Unger R. Machine learning models predict 30-day and 1-year mortality in heart failure. J Am Coll Cardiol. 2020. https://doi.org/10.1016/s07351097(20)31485-6

Publisher's Note Springer Nature remains neutral with regard to jurisdictional claims in published maps and institutional affiliations. 